\title{
A vine copula mixed effect model for trivariate meta-analysis of diagnostic test accuracy studies accounting for disease prevalence
}

\author{
Aristidis K. Nikoloulopoulos*
}

\begin{abstract}
A bivariate copula mixed model has been recently proposed to synthesize diagnostic test accuracy studies and it has been shown that is superior to the standard generalized linear mixed model (GLMM) in this context. Here we call trivariate vine copulas to extend the bivariate meta-analysis of diagnostic test accuracy studies by accounting for disease prevalence. Our vine copula mixed model includes the trivariate GLMM as a special case and can also operate on the original scale of sensitivity, specificity, and disease prevalence. Our general methodology is illustrated by reanalysing the data of two published meta-analyses. Our study suggests that there can be an improvement on trivariate GLMM in fit to data and makes the argument for moving to vine copula random effects models especially because of their richness including reflection asymmetric tail dependence, and, computational feasibility despite their three-dimensionality.
\end{abstract}

Key Words: copula models; diagnostic tests; multivariate meta-analysis; random effects models; sensitivity/specificity/prevalence; vines.

\section{Introduction}

Synthesis of diagnostic test accuracy studies is the most common medical application of multivariate meta-analysis. ${ }^{1-3}$ Diagnostic test accuracy studies observe the result of a gold standard procedure which defines the presence or absence of a disease and the result of a diagnostic test. They typically report the number of true positives (diseased people correctly diagnosed), false positives (non-diseased people incorrectly diagnosed as diseased), true negatives and false negatives.

In situations where studies compare a diagnostic test with its gold standard, heterogeneity arises between studies due to the differences in disease prevalence, study design as well as laboratory and other characteristics. ${ }^{4}$ Because of this heterogeneity, a generalized linear mixed model (GLMM) has been recommended in the biostatistics literature to synthesize information. ${ }^{3,5-7}$ The GLMM assumes

${ }^{*}$ A.Nikoloulopoulos@uea.ac.uk, School of Computing Sciences, University of East Anglia, Norwich Research Park, Norwich NR4 7TJ, UK 
independent binomial distributions for the true positives and true negatives, conditional on the latent pair of transformed (via a link function) sensitivity and specificity in each study. The random effects (latent pair of transformed sensitivity and specificity) are jointly analysed with a bivariate normal (BVN) distribution.

However, it is reported in the literature that sensitivity and specificity may not reflect the clinical utility of a diagnostic test; such clinical utility depends on the prevalence of disease in the population to which the diagnostic test is applied. ${ }^{8}$ Furthermore, many empirical studies have shown the assumption of independence between the sensitivity/specificity with disease prevalence for a dichotomous disease status is likely to be violated. ${ }^{9-11}$

To this end, Chu et al. ${ }^{8}$ extended the bivariate GLMM to a trivariate GLMM by also accounting for disease prevalence. In the same manner, the trivariate GLMM assumes independent binomial distributions for the true positives, true negatives, and diseased persons conditional on a latent vector of transformed (via a link function) sensitivity, specificity, and prevalence in each study. The random effects herein are jointly analysed with a trivariate normal (TVN) distribution.

In this paper we propose a vine copula mixed model as an extension of the trivariate GLMM by rather using a vine copula representation of the random effects distribution with normal and beta margins. Our general model (a) includes the trivariate GLMM as a special case, (b) can also operate on the original scale of sensitivity, specificity, and prevalence, and (c) can also provide tail dependencies and asymmetries.

In fact, we extend the copula mixed model proposed by Nikoloulopoulos ${ }^{12}$ to the trivariate case accounting for disease prevalence. There are many simple bivariate copula families, but generally their multivariate extensions have limited dependence structures. For example, Archimedean copulas in more that two dimensions allow only exchangeable structure with a narrower range of negative dependence as the dimension increases. ${ }^{13,14}$ The multivariate normal (MVN) copula generated by the MVN distribution inherits the useful properties of the latter, thus allowing a wide range for dependence, and overcomes the drawback of limited dependence inherent in simple parametric families of copulas. ${ }^{15}$ However, it provides tail independence and reflection symmetry, thus it is not suitable when data display dependence among extreme values and inferences based on multivariate tail probabilities are needed. ${ }^{16}$ In Nikoloulopoulos ${ }^{12}$ it has shown that such asymmetries are expected in binary data from diagnostic accuracy test studies and summary receiver operating characteristic inference is particularly based on bivariate tail probabilities.

In recent years, a popular and useful approach is the vine pair-copula construction which is based on $d(d-1) / 2$ bivariate copulas, of which some are used to summarize conditional dependence; a special case occurs if all of these bivariate copulas are BVN, and then the parametrization of an MVN copula is a set of correlations and partial correlations that are algebraically independent in $(-1,1)^{d(d-1) / 2} \cdot{ }^{17,18}$ 
The $d$-dimensional vine copulas can cover flexible dependence structures through the specification of $d-1$ bivariate marginal copulas at level 1 and $(d-1)(d-2) / 2$ bivariate conditional copulas at higher levels; at level $\ell$ for $\ell=2, \ldots, d-1$, there are $d-\ell$ bivariate conditional copulas that condition on $\ell-1$ variables. Vine copulas include MVN as special case, but can also cover reflection asymmetry and have upper/lower tail dependence parameters being different for each bivariate margin $(i, j)$. Joe et al. ${ }^{16}$ have a main theorem that says that all bivariate margins of the vine copula have upper/lower tail dependence if the bivariate copulas at level 1 have upper/lower tail dependence.

A vine copula approach for meta-analysis of diagnostic accuracy studies was recently proposed by Hoyer and $\mathrm{Kuss}^{19}$ who explored the use of a trivariate vine copula model for observed discrete variables (number of true positives, true negatives, and diseased persons) which have beta-binomial margins. This model is actually an approximation of the vine copula mixed model with beta margins for the latent vector of sensitivity, specificity, and prevalence. Nikoloulopoulos ${ }^{12}$ has extensively studied the small-sample and theoretical efficiency of this approximation in the bivariate case. ${ }^{20}$ It was clearly shown that this approximation is an inefficient approach; it leads to substantial downward bias for the estimates of the dependence and bias for the meta-analytic parameters for fully specified copula mixed models. This evolves because there are serious problems on modelling assumptions under the case of heterogeneous study sizes. If the number of true positives, true negatives, and diseased persons do not have a common support over different studies, then one cannot conclude that there is a copula.

Furthermore, Hoyer and Kuss ${ }^{19}$ compared fits and inference for vine copulas, when the bivariate copulas are all reflection symmetric, i.e., Placket or BVN. In this paper, we make the first use of vine copulas with bivariate linking copulas that can have upper tail dependence different from lower tail dependence. This provides a means to check if there is some reflection asymmetry in the joint tails of diagnostic accuracy data from binary test outcomes.

The remainder of the paper proceeds as follows. Section 2 summarizes the standard GLMM for synthesis of diagnostic test accuracy studies accounting for disease prevalence. Section 3 has a brief overview of relevant vine copula theory and then introduces the vine copula mixed model for diagnostic test accuracy studies accounting for disease prevalence, discusses its relationship with existing models, and provides computational details for maximum likelihood estimation. Section 4 discusses practical issues for (trivariate) vine copula modelling that have been neglected in Hoyer and Kuss ${ }^{19}$. Section 5 contains small-sample efficiency calculations to investigate the effect of misspecifying the random effects distribution on parameter estimators and standard errors. Section 6 presents applications of our methodology to two data frames with diagnostic accuracy data from binary test outcomes. We conclude with some discussion in Section 7 , followed by a brief section with the software details. 


\section{The standard GLMM}

We first introduce the notation used in this paper. The focus is on two-level (within-study and between-studies) cluster data. The data are $\left(y_{i j}, n_{i j}\right), i=1, \ldots, N, j=1,2,3$, where $j$ is an index for the within study measurements and $i$ is an index for the individual studies.

The standard two-level model of meta-analysing diagnostic test accuracy studies accounting for disease prevalence ${ }^{3,8}$ lies in the framework of mixed models ${ }^{21}$. The within-study model assumes that the number of true positives $Y_{i 1}$, true negatives $Y_{i 2}$, and diseased persons $Y_{i 3}$ are conditionally independent and binomially distributed given $\mathbf{X}=\mathbf{x}$, where $\mathbf{X}=\left(X_{1}, X_{2}, X_{3}\right)$ denotes the trivariate latent (random) vector of transformed sensitivity, specificity, and disease prevalence. That is

$$
\begin{aligned}
& Y_{i 1} \mid X_{1}=x_{1} \sim \operatorname{Binomial}\left(n_{i 1}, l^{-1}\left(x_{1}\right)\right) ; \\
& Y_{i 2} \mid X_{2}=x_{2} \sim \operatorname{Binomial}\left(n_{i 2}, l^{-1}\left(x_{2}\right)\right) ; \\
& Y_{i 3} \mid X_{3}=x_{3} \sim \operatorname{Binomial}\left(n_{i 3}, l^{-1}\left(x_{3}\right)\right),
\end{aligned}
$$

where $l(\cdot)$ is a link function such as the commonly used logit. The between studies model assumes that $\mathbf{X}$ is TVN distributed with mean vector $\boldsymbol{\mu}=\left(l\left(\pi_{1}\right), l\left(\pi_{2}\right), l\left(\pi_{3}\right)\right)^{\top}$ and variance covariance matrix $\boldsymbol{\Sigma}=\left(\begin{array}{ccc}\sigma_{1}^{2} & \rho_{12} \sigma_{1} \sigma_{2} & \rho_{13} \sigma_{1} \sigma_{3} \\ \rho_{12} \sigma_{1} \sigma_{2} & \sigma_{2}^{2} & \rho_{23} \sigma_{2} \sigma_{3} \\ \rho_{13} \sigma_{1} \sigma_{3} & \rho_{23} \sigma_{2} \sigma_{3} & \sigma_{3}^{2}\end{array}\right)$. That is

$$
\mathbf{X} \sim \operatorname{TVN}(\boldsymbol{\mu}, \boldsymbol{\Sigma})
$$

The models in (1) and (2) together specify a GLMM with joint likelihood

$$
L\left(\pi_{1}, \pi_{2}, \pi_{3}, \sigma_{1}, \sigma_{2}, \sigma_{3}, \rho_{12}, \rho_{13}, \rho_{23}\right)=\prod_{i=1}^{N} \iint \prod_{j=1}^{3} g\left(y_{i j} ; n_{i j}, l^{-1}\left(x_{j}\right)\right) \phi_{123}\left(x_{1}, x_{2}, x_{3} ; \boldsymbol{\mu}, \boldsymbol{\Sigma}\right) d x_{1} d x_{2} d x_{3},
$$

where

$$
g(y ; n, \pi)=\left(\begin{array}{l}
n \\
y
\end{array}\right) \pi^{y}(1-\pi)^{n-y}, \quad y=0,1, \ldots, n, \quad 0<\pi<1,
$$

is the binomial probability mass function $(\mathrm{pmf})$ and $\phi_{123}(\cdot ; \boldsymbol{\mu}, \boldsymbol{\Sigma})$ is the TVN density with mean vector $\boldsymbol{\mu}$ and variance covariance matrix $\boldsymbol{\Sigma}$. The parameters $\pi_{1}, \pi_{2}$ and $\pi_{3}$ are those of actual interest denoting the meta-analytic parameters for the sensitivity, specificity, and disease prevalence, respectively, while the univariate parameters $\sigma_{1}^{2}, \sigma_{2}^{2}$, and $\sigma_{3}^{2}$ are of secondary interest denoting the variability between studies.

\section{The vine copula mixed model for diagnostic test accuracy studies}

In this section, we introduce the vine copula mixed model for diagnostic test accuracy studies and discuss its relationship with existing models. Before that, the first subsection has some background 
on copula models. In Subsection 3.2 and Subsection 3.3 a vine copula representation of the random effects distribution with normal and beta margins respectively is presented. We complete this section with details on maximum likelihood estimation.

\subsection{Overview and relevant background for vine copulas}

A copula is a multivariate cdf with uniform $U(0,1)$ margins. ${ }^{13,18,22}$ If $F$ is a $d$-variate cdf with univariate margins $F_{1}, \ldots, F_{d}$, then Sklar's ${ }^{23}$ theorem implies that there is a copula $C$ such that

$$
F\left(x_{1}, \ldots, x_{d}\right)=C\left(F_{1}\left(x_{1}\right), \ldots, F_{d}\left(x_{d}\right)\right)
$$

The copula is unique if $F_{1}, \ldots, F_{d}$ are continuous. If $F$ is continuous and $\left(Y_{1}, \ldots, Y_{d}\right) \sim F$, then the unique copula is the distribution of $\left(U_{1}, \ldots, U_{d}\right)=\left(F_{1}\left(Y_{1}\right), \ldots, F_{d}\left(Y_{d}\right)\right)$ leading to

$$
C\left(u_{1}, \ldots, u_{d}\right)=F\left(F_{1}^{-1}\left(u_{1}\right), \ldots, F_{d}^{-1}\left(u_{d}\right)\right), \quad 0 \leq u_{j} \leq 1, j=1, \ldots, d
$$

where $F_{j}^{-1}$ are inverse cdfs. ${ }^{24}$ In particular, if $\Phi_{d}(\cdot ; \mathbf{R})$ is the MVN cdf with correlation matrix

$$
\mathbf{R}=\left(\rho_{j k}: 1 \leq j<k \leq d\right)
$$

and $\mathrm{N}(0,1)$ margins, and $\Phi$ is the univariate standard normal cdf, then the MVN copula is

$$
C\left(u_{1}, \ldots, u_{d}\right)=\Phi_{d}\left(\Phi^{-1}\left(u_{1}\right), \ldots, \Phi^{-1}\left(u_{d}\right) ; \mathbf{R}\right) .
$$

A copula $C$ has reflection symmetry if $\left(U_{1}, \ldots, U_{d}\right) \sim C$ implies that $\left(1-U_{1}, \ldots, 1-U_{d}\right)$ has the same distribution $C$. This is apparently the case for the MVN copula. When it is necessary to have copula models with reflection asymmetry and flexible lower/upper tail dependence, then vine copulas are the best choice. ${ }^{16}$ The $d$-dimensional vine copulas are built via successive mixing from $d(d-1) / 2$ bivariate linking copulas on trees and their cdfs involve lower-dimensional integrals. Since the densities of multivariate vine copulas can be factorized in terms of bivariate linking copulas and lower-dimensional margins, they are computationally tractable. Depending on the types of trees, various vine copulas can be constructed. Two types are D-vines and C-vines.

For the $d$-dimensional D-vine, the pairs at level 1 are $i, i+1$, for $i=1, \ldots, d-1$, and for level $\ell(2 \leq \ell<d)$, the (conditional) pairs are $i, i+\ell \mid i+1, \ldots, i+\ell-1$ for $i=1, \ldots, d-\ell$. For the $d$-dimensional $\mathrm{C}$-vine, the pairs at level 1 are $1, i$, for $i=2, \ldots, d$, and for level $\ell(2 \leq \ell<d)$, the (conditional) pairs are $\ell, i \mid 1, \ldots, \ell-1$ for $i=\ell+1, \ldots, d$. That is, for the $\mathrm{D}$-vine, conditional copulas are specified for variables $i$ and $i+\ell$ given the variables indexed in between; and for the $\mathrm{C}$-vine, conditional copulas are specified for variables $\ell$ and $i$ given those indexed as 1 to $\ell-1 .^{25}$ In Figures 1 and 2, a D-vine and a C-vine with 3 variables and 2 trees/levels are depicted. From the graphs 
is apparent that the $\mathrm{D}$ - and $\mathrm{C}$-vine structures in the three-dimensional case coincide. The trivariate density is decomposed in a simple manner by multiplying the nodes of the nested set of trees

$$
\begin{aligned}
f_{123}\left(x_{1}, x_{2}, x_{3}\right) & =f_{2}\left(x_{2}\right) f_{1}\left(x_{1}\right) f_{3}\left(x_{3}\right) c_{12}\left(F_{1}\left(x_{1}\right), F_{2}\left(x_{2}\right)\right) c_{13}\left(F_{1}\left(x_{1}\right), F_{3}\left(x_{3}\right)\right) c_{23 \mid 1}\left(F_{2 \mid 1}\left(x_{2} \mid x_{1}\right), F_{3 \mid 1}\left(x_{3} \mid x_{1}\right)\right) \\
& =f_{1}\left(x_{1}\right) f_{2}\left(x_{2}\right) f_{3}\left(x_{3}\right) c_{123}\left(F_{1}\left(x_{1}\right), F_{2}\left(x_{2}\right), F_{3}\left(x_{3}\right)\right)
\end{aligned}
$$

where $F_{j \mid k}\left(x_{j} \mid x_{k}\right)=\partial C_{j k}\left(F_{j}\left(x_{j}\right), F_{k}\left(x_{k}\right)\right) / \partial F_{k}\left(x_{k}\right) .{ }^{26}$

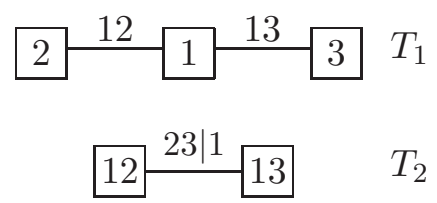

Figure 1: 3 -dimensional D-vine with 2 trees/levels $\left(T_{j}, j=1,2\right)$.

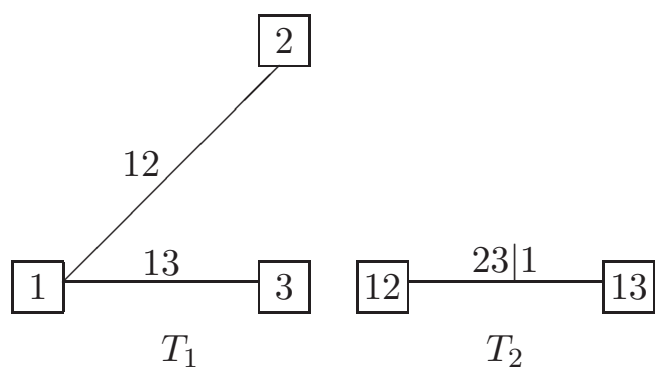

Figure 2: 3-dimensional C-vine with 2 trees/levels $\left(T_{j}, j=1,2\right)$ where the dependence is driven by $Y_{1}$.

\subsection{The copula mixed model for the latent pair of transformed sensitivity and specificity}

Here we generalize the GLMM by proposing a model that links the three random effects using a copula function rather than the TVN distribution.

The within-study model is the same as in the standard GLMM; see (1). The stochastic representation of the between studies model takes the form

$$
\left(\Phi\left(X_{1} ; l\left(\pi_{1}\right), \sigma_{1}^{2}\right), \Phi\left(X_{2} ; l\left(\pi_{2}\right), \sigma_{2}^{2}\right), \Phi\left(X_{3} ; l\left(\pi_{3}\right), \sigma_{3}^{2}\right)\right) \sim C(\cdot ; \boldsymbol{\theta}),
$$

where $C(\cdot ; \boldsymbol{\theta})$ is a vine copula with dependence parameter vector $\boldsymbol{\theta}=\left(\theta_{12}, \theta_{13}, \theta_{23 \mid 1}\right)$ and $\Phi\left(\cdot ; \mu, \sigma^{2}\right)$ is the cdf of the $\mathrm{N}\left(\mu, \sigma^{2}\right)$ distribution. The joint density $f_{123}\left(x_{1}, x_{2}, x_{3}\right)$ of the transformed latent proportions can be derived using the vine density representation in (4):

$$
\begin{aligned}
& f_{123}\left(x_{1}, x_{2}, x_{3} ; \pi_{1}, \pi_{2}, \pi_{3}, \sigma_{1}, \sigma_{2}, \sigma_{3}, \boldsymbol{\theta}\right)= \\
& c_{123}\left(\Phi\left(x_{1} ; l\left(\pi_{1}\right), \sigma_{1}^{2}\right), \Phi\left(x_{2} ; l\left(\pi_{2}\right), \sigma_{2}^{2}\right), \Phi\left(x_{3} ; l\left(\pi_{3}\right), \sigma_{3}^{2}\right) ; \boldsymbol{\theta}\right) \prod_{j=1}^{3} \phi\left(x_{j} ; l\left(\pi_{j}\right), \sigma_{j}^{2}\right),
\end{aligned}
$$


where $\phi\left(\cdot ; \mu, \sigma^{2}\right)$ is the $\mathrm{N}\left(\mu, \sigma^{2}\right)$ density. The models in (1) and (5) together specify a vine copula mixed model with joint likelihood

$$
\begin{aligned}
L\left(\pi_{1}, \pi_{2}, \sigma_{1}, \sigma_{2}, \boldsymbol{\theta}\right) & =\prod_{i=1}^{N} \int_{-\infty}^{\infty} \int_{-\infty}^{\infty} \int_{-\infty}^{\infty} \prod_{j=1}^{3} g\left(y_{i j} ; n_{i j}, l^{-1}\left(x_{j}\right)\right) c_{123}\left(\Phi\left(x_{1} ; l\left(\pi_{1}\right), \sigma_{1}^{2}\right), \Phi\left(x_{2} ; l\left(\pi_{2}\right),\right.\right. \\
& \left.\left.\sigma_{2}^{2}\right) \Phi\left(x_{3} ; l\left(\pi_{3}\right), \sigma_{3}^{2}\right) ; \boldsymbol{\theta}\right) \prod_{j=1}^{3} \phi\left(x_{j} ; l\left(\pi_{j}\right), \sigma_{j}^{2}\right) d x_{j} \\
& =\prod_{i=1}^{N} \int_{0}^{1} \int_{0}^{1} \int_{0}^{1} \prod_{j=1}^{3} g\left(y_{i j} ; n_{i j}, l^{-1}\left(\Phi^{-1}\left(u_{j} ; l\left(\pi_{j}\right), \sigma_{j}^{2}\right)\right)\right) c_{123}\left(u_{1}, u_{2}, u_{3} ; \boldsymbol{\theta}\right) d u_{j}
\end{aligned}
$$

\subsubsection{Relationship with the GLMM}

In this subsection, we show what happens when the bivariate copula block is the BVN copula. The re-

sulting distribution is the TVN with variance-covariance matrix $\boldsymbol{\Sigma}$, where $\rho_{23}=\rho_{23 \mid 1} \sqrt{1-\rho_{12}^{2}} \sqrt{1-\rho_{13}^{2}}+$ $\rho_{12} \rho_{13} \cdot{ }^{27}$ In fact, the correlation parameter $\rho_{23 \mid 1}$ of $c_{23 \mid 1}$, is the partial correlation of $X_{2}$ and $X_{3}$ given $X_{1}$.

\subsection{The vine copula mixed model for the latent pair of sensitivity and specificity}

The within-study model assumes that the number of true positives $Y_{i 1}$, true negatives $Y_{i 2}$, and diseased persons $Y_{i 3}$ are conditionally independent and binomially distributed given $\mathbf{X}=\mathbf{x}$, where $\mathbf{X}=\left(X_{1}, X_{2}, X_{3}\right)$ denotes the bivariate latent random pair of sensitivity and specificity. That is

$$
\begin{aligned}
Y_{i 1} \mid X_{1}=x_{1} & \sim \operatorname{Binomial}\left(n_{i 1}, x_{1}\right) \\
Y_{i 2} \mid X_{2}=x_{2} & \sim \operatorname{Binomial}\left(n_{i 2}, x_{2}\right) \\
Y_{i 3} \mid X_{3}=x_{3} & \sim \operatorname{Binomial}\left(n_{i 3}, x_{3}\right) .
\end{aligned}
$$

So one does not have to transform the latent sensitivity and specificity and can work on the original scale. The $\operatorname{Beta}(\alpha, \beta)$ distribution can be used for the marginal modeling of the latent proportions and its density is

$$
f(x ; \alpha, \beta)=\frac{x^{\alpha-1}(1-x)^{\beta-1}}{B(\alpha, \beta)}, \quad 0<x<1, \quad \alpha, \beta>0 .
$$

In the sequel we will use the $\operatorname{Beta}(\pi, \gamma)$ parametrization, where $\pi=\frac{\alpha}{\alpha+\beta}$ (mean parameter) and $\gamma=\frac{1}{\alpha+\beta+1}$ (dispersion parameter).

The stochastic representation of the between studies model is

$$
\left(F\left(X_{1} ; \pi_{1}, \gamma_{1}\right), F\left(X_{2} ; \pi_{2}, \gamma_{2}\right), F\left(X_{3} ; \pi_{3}, \gamma_{3}\right)\right) \sim C(\cdot ; \boldsymbol{\theta}),
$$


where $C(\cdot ; \boldsymbol{\theta})$ is a vine copula with dependence parameter vector $\boldsymbol{\theta}$ and $F(\cdot ; \pi, \gamma)$ is the cdf of the the $\operatorname{Beta}(\pi, \gamma)$ distribution. The models in (8) and (9) together specify a vine copula mixed model with joint likelihood

$$
\begin{aligned}
L\left(\pi_{1}, \pi_{2}, \pi_{3}, \gamma_{1}, \gamma_{2}, \gamma_{3}, \boldsymbol{\theta}\right) & \left.=\prod_{i=1}^{N} \int_{0}^{1} \int_{0}^{1} \int_{0}^{1} \prod_{j=1}^{3} g\left(y_{i j} ; n_{i j}, x_{j}\right) ; \boldsymbol{\theta}\right) \prod_{123}\left(F\left(x_{1} ; \pi_{1}, \gamma_{1}\right), F\left(x_{j} ; \pi_{2}, \gamma_{2}\right), F\left(x_{j}, \gamma_{j}\right) d x_{j}\right. \\
& =\prod_{i=1}^{N} \int_{0}^{1} \int_{0}^{1} \int_{0}^{1} \prod_{j=1}^{3} g\left(y_{i j} ; n_{i j}, F^{-1}\left(u_{j} ; \pi_{j}, \gamma_{j}\right)\right) c\left(u_{1}, u_{2}, u_{3} ; \boldsymbol{\theta}\right) d u_{j}
\end{aligned}
$$

In the vine copula mixed model with beta (normal) margins, it is important to note that the copula parameter vector $\boldsymbol{\theta}$ contains parameters of the random effects model and it is separated from the univariate parameters. The univariate parameters $\pi_{j}$ 's are those of actual interest denoting the meta-analytic parameters for the sensitivity, specificity, and disease prevalence, while the univariate parameters $\sigma_{j}$ 's $\left(\gamma_{j}\right.$ 's) are of secondary interest expressing the variability between studies.

\subsubsection{Relationship with existing models}

Hoyer and Kuss ${ }^{19}$ proposed a vine copula model with beta-binomial margins in this context. This model is actually an approximation of the copula mixed model with beta margins for the latent pair of sensitivity and specificity in (8) and (9). They attempt to approximate the likelihood in (10) with the likelihood of a copula model for observed discrete variables which have beta-binomial margins.

The approximation that they suggest is

$$
\begin{aligned}
& L\left(\pi_{1}, \pi_{2}, \pi_{3}, \gamma_{1}, \gamma_{2}, \gamma_{3}, \boldsymbol{\theta}\right) \approx \\
& \prod_{i=1}^{N} c_{123}\left(H\left(y_{i 1} ; n_{i 1}, \pi_{1}, \gamma_{1}\right), H\left(y_{i 2} ; n_{i 2}, \pi_{2}, \gamma_{2}\right), H\left(y_{i 3} ; n_{i 3}, \pi_{3}, \gamma_{3}\right) ; \boldsymbol{\theta}\right) \prod_{j=1}^{3} h\left(y_{i j} ; n_{i j}, \pi_{j}, \gamma_{j}\right),
\end{aligned}
$$

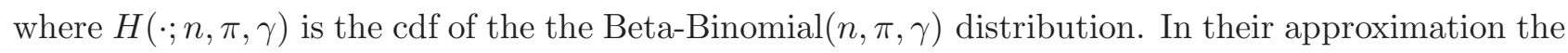
authors also treat the observed variables which have beta-binomial distributions as being continuous, and model them under the theory for copula models with continuous margins. They state that the computations are becoming too complex in the trivariate case. This is far from true; for $d=3$ the evaluation of the discrete likelihood can be handled extremely easily. Copula modelling for $d$ dimensional discrete data is studied by Nikoloulopoulos ${ }^{28,29}$ for MVN copulas and by Panagiotelis et $a l .{ }^{30}$ and Nikoloulopoulos and $\mathrm{Joe}^{24}$ for vine copulas for a dimension $d$ much higher than 3.

The main problem in Hoyer and Kuss ${ }^{19}$ (even if treating the observed variables which have betabinomial distributions as being discrete) is that the discrete $Y_{i j}$ do not have a common support 
over different studies or $i$, hence, one cannot conclude that there is a copula for $\left(Y_{i 1}, Y_{i 2}, Y_{i 3}\right)$ that applies when the $n_{i j}$ vary with different studies $i$. The natural replicability is in the random effects probability for sensitivity, specificity, and disease prevalence. Nikoloulopoulos ${ }^{12}$ has shown in the bivariate case that this approximation ${ }^{20}$ is an inefficient approach; it leads to substantial downward bias for the estimates of the copula parameters and bias for the meta-analytic parameters for fully specified copula mixture models.

Therefore, the approximation method in Hoyer and Kuss ${ }^{19}$ is not discussed/used in the sequel since its inefficiency with theoretical and small-sample efficiency calculations has been proven ${ }^{12}$ and should be avoided for meta-analysis of diagnostic accuracy test studies.

\subsection{Maximum likelihood estimation and computational details}

Estimation of the model parameters $\left(\pi_{1}, \pi_{2}, \pi_{3}, \sigma_{1}, \sigma_{2}, \sigma_{3}, \boldsymbol{\theta}\right)$ and $\left(\pi_{1}, \pi_{2}, \pi_{3}, \gamma_{1}, \gamma_{2}, \gamma_{3}, \boldsymbol{\theta}\right)$ can be approached by the standard maximum likelihood (ML) method, by maximizing the logarithm of the joint likelihood in (7) and (10), respectively. The estimated parameters can be obtained by using a quasi-Newton ${ }^{31}$ method applied to the logarithm of the joint likelihood. This numerical method requires only the objective function, i.e., the logarithm of the joint likelihood, while the gradients are computed numerically and the Hessian matrix of the second order derivatives is updated in each iteration. The standard errors (SE) of the ML estimates can be also obtained via the gradients and the Hessian computed numerically during the maximization process. Assuming that the usual regularity conditions for asymptotic maximum likelihood theory hold for the trivariate model as well as for its margins we have that ML estimates are asymptotically normal. ${ }^{32}$ Therefore one can build Wald tests to statistically judge any effect.

For vine copula mixed models of the form with joint likelihood as in (7) and (10), numerical evaluation of the joint pmf is easily done with the following steps:

1. Calculate Gauss-Legendre ${ }^{33}$ quadrature points $\left\{u_{q}: q=1, \ldots, n_{q}\right\}$ and weights $\left\{w_{q}: q=\right.$ $\left.1, \ldots, n_{q}\right\}$ in terms of standard uniform.

2. Convert from independent uniform random variables $\left\{u_{q_{1}}: q_{1}=1, \ldots, n_{q}\right\},\left\{u_{q_{2}}: q_{2}=\right.$ $\left.1, \ldots, n_{q}\right\}$, and $\left\{u_{q_{3}}: q_{3}=1, \ldots, n_{q}\right\}$ to dependent uniform random variables $\left\{v_{q_{1}}=u_{q_{1}}: q_{1}=\right.$ $\left.1, \ldots, n_{q}\right\},\left\{v_{q_{2} \mid q_{1}}=C_{12}^{-1}\left(u_{q_{2}} \mid u_{q_{1}} ; \theta_{12}\right): q_{1}=q_{2}=1, \ldots, n_{q}\right\}$, and $\left\{v_{q_{2} q_{3} \mid q_{1}}=C_{13}^{-1}\left(C_{23 \mid 1}^{-1}\left(u_{q_{3}} \mid u_{q_{2}} ;\right.\right.\right.$ $\left.\left.\left.\theta_{23 \mid 1}\right) \mid u_{q_{1}} ; \theta_{13}\right): q_{1}=q_{2}=q_{3}=1, \ldots, n_{q}\right\}$ that have vine distribution $C(\cdot ; \boldsymbol{\theta})$. The inverse of the conditional distribution $C(v \mid u ; \theta)=\partial C(u, v ; \theta) / \partial u$ corresponding to the copula $C(\cdot ; \theta)$ and the simulation algorithm of a C-vine copula in $\mathrm{Joe}^{34}$ are used to achieve this. 
3. Numerically evaluate the joint pmf, e.g.,

$$
\int_{0}^{1} \int_{0}^{1} \prod_{j=1}^{3} g\left(y_{j} ; n_{j}, F^{-1}\left(u_{j} ; \pi_{j}, \gamma_{j}\right)\right) c\left(u_{1}, u_{2}, u_{3} ; \boldsymbol{\theta}\right) d u_{1} d u_{2} d u_{3}
$$

in a triple sum:

$\sum_{q_{1}=1}^{n_{q}} \sum_{q_{2}=1}^{n_{q}} \sum_{q_{3}=1}^{n_{q}} w_{q_{1}} w_{q_{2}} w_{q_{3}} g\left(y_{1} ; n, F^{-1}\left(v_{q_{1}} ; \pi_{1}, \gamma_{1}\right)\right) g\left(y_{2} ; n, F^{-1}\left(v_{q_{2} \mid q_{1}} ; \pi_{2}, \gamma_{2}\right)\right) g\left(y_{3} ; n, F^{-1}\left(v_{q_{2} q_{3} \mid q_{1}} ; \pi_{3}, \gamma_{3}\right)\right)$.

With Gauss-Legendre quadrature, the same nodes and weights are used for different functions; this helps in yielding smooth numerical derivatives for numerical optimization via quasi-Newton. ${ }^{31}$ Our comparisons show that $n_{q}=15$ is adequate with good precision to at least at four decimal places; thus it also provides the advantage of fast implementation. To this end, the vine copula mixed effect model for meta-analysis of diagnostic test accuracy studies with a triple integral is in fact straightforward computationally.

\section{Construction of vine copulas}

A vine copula can be decomposed into a sequence of bivariate conditional copulas in many ways if the conditional copulas depend on the values of the conditioning variables. Vine copulas involve a sequence of conditional bivariate copulas, but assume the bivariate conditional copulas are constant over the conditioning variables. For example, MVN copulas satisfy this property with conditional correlations being equal to partial correlations. In general, a given multivariate copula might be wellapproximated by several different vine copulas, and likelihood inference could be used to find such vines. ${ }^{25}$

Vine copulas can provide a wide range of flexible (tail) dependence by choosing suitable choices of bivariate linking copulas, ${ }^{16}$ but they require a decision on the indexing of variables since for a 3 dimensional C-vine or D-vine copula there are 3!/2 possible different choices (distinct permutations).

In this section, we discuss the following practical issues for trivariate vine-copula modelling:

1. type of bivariate copula families as building blocks;

2. the matching of variables to labels/indexes;

3. conditional independence.

These matters have been overseen in Hoyer and $\mathrm{Kuss}^{19}$.

\subsection{Building blocks}

For fitting trivariate data with possible tail dependence (in which case, TVN copulas are inappropriate), we use vine copulas with parametric families for the bivariate building blocks that have different strengths of tail behaviour. ${ }^{12}$ 
- Reflection symmetric copulas with tail independence satisfying $C(u, u)=O\left(u^{2}\right)$ and $\bar{C}(1-u, 1-$ $u)=O\left(u^{2}\right)$ as $u \rightarrow 0$, such as the Frank copula with inverse conditional cdf

$$
C^{-1}(v \mid u ; \theta)=-\frac{1}{\theta} \log \left[\frac{1-\left(1-e^{-\theta}\right)}{\left(v^{-1}-1\right) e^{-\theta u}+1}\right], \quad \theta \in(-\infty, \infty) \backslash\{0\} .
$$

- Reflection symmetric copulas with intermediate tail dependence ${ }^{35}$ such as the BVN copula, which satisfies $C(u, u, \theta)=O\left(u^{2 /(1+\theta)}(-\log u)^{-\theta /(1+\theta)}\right)$ as $u \rightarrow 0$ with inverse conditional cdf

$$
C^{-1}(v \mid u ; \theta)=\Phi\left(\sqrt{1-\theta^{2}} \Phi^{-1}(v)+\theta \Phi^{-1}(u)\right), \quad \theta \in[-1,1] .
$$

- Reflection asymmetric copulas with lower tail dependence satisfying $c(u, u)=O\left(u^{-1}\right)$ as $u \rightarrow 0$, such as the Clayton copula with inverse conditional cdf

$$
C^{-1}(v \mid u ; \theta)=\left\{\left(v^{-\theta /(1+\theta)}-1\right) u^{-\theta}+1\right\}^{-1 / \theta}, \quad \theta \in(0, \infty) .
$$

- Reflection asymmetric copulas with upper tail dependence satisfying $c(1-u, 1-u)=O\left(u^{-1}\right)$ as $u \rightarrow 0$, such as the rotated by 180 degrees Clayton copula with inverse conditional cdf

$$
C^{-1}(v \mid u ; \theta)=1-\left[\left\{(1-v)^{-\theta /(1+\theta)}-1\right\}(1-u)^{-\theta}+1\right]^{-1 / \theta}, \quad \theta \in(0, \infty) .
$$

- Reflection asymmetric copula family with negative upper-lower tail dependence satisfying $c(1-$ $u, u)=O\left(u^{-1}\right)$ as $u \rightarrow 0$, such as the rotated by 90 degrees Clayton copula with inverse conditional cdf

$$
C^{-1}(v \mid u ; \theta)=\left\{\left(v^{\theta /(1-\theta)}-1\right)(1-u)^{\theta}+1\right\}^{1 / \theta}, \quad \theta \in(0, \infty)
$$

- Reflection asymmetric copula family with negative lower-upper tail dependence satisfying $c(u, 1-$ $u)=O\left(u^{-1}\right)$ as $u \rightarrow 0$, such as the as the rotated by 270 degrees Clayton copula with inverse conditional cdf

$$
C^{-1}(v \mid u ; \theta)=1-\left[\left\{(1-v)^{\theta /(1-\theta)}-1\right\} u^{\theta}+1\right]^{1 / \theta}, \quad \theta \in(0, \infty) .
$$

The above copula families are sufficient for the applications in Section 6, since tail dependence is a property to consider when choosing amongst different families of copulas and the concept of upper/lower tail dependence is one way to differentiate families. Nikoloulopoulos and Karlis ${ }^{36}$ have shown that it is hard to choose a copula with similar properties from real data, since copulas with similar (tail) dependence properties provide similar fit. Hoyer and Kuss ${ }^{19}$ used, also, the Placket copula; this bivariate block is not used here since we have included another choice of copulas with similar properties i.e., the Frank parametric family of bivariate copulas. 


\subsection{Indexing of the variables}

The next step would be to decide on the bivariate pairs to put at level 1 of the vine. Given the small dimension $(d=3)$ we can fit all possible permutations of the vine and select the best based on the likelihood principle. In Figures 2, 3, and 4 we depict the three distinct permutations:

$$
\{12,13,23 \mid 1\}, \quad\{12,23,13 \mid 2\}, \quad \text { and } \quad\{13,23,12 \mid 3\}
$$

using a C-vine structure.

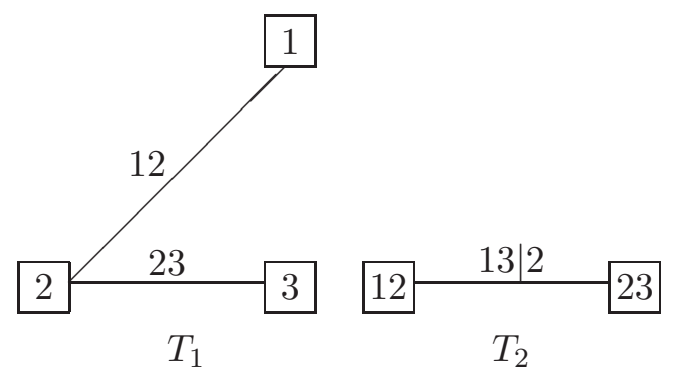

Figure 3: 3-dimensional C-vine with 2 trees/levels $\left(T_{j}, j=1,2\right)$ where the dependence is driven by $Y_{2}$.

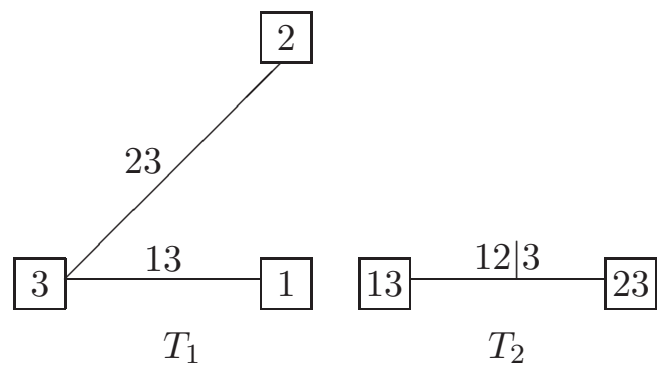

Figure 4: 3-dimensional C-vine with 2 trees/levels $\left(T_{j}, j=1,2\right)$ where the dependence is driven by $Y_{3}$.

\subsection{Conditional independence}

Joe et al. ${ }^{16}$ show that in order for a vine copula to have (tail) dependence for all bivariate margins, it is only necessary for the bivariate copulas in level 1 to have (tail) dependence and it is not necessary for the conditional bivariate copulas in levels $2, \ldots, d-1$ to have tail dependence. That provides the theoretical justification for the idea to capture the strongest dependence in the first tree and then just use the independence copulas in lower order tree(s), i.e., conditional independence.

The improvement over the reduction of the dependence parameters, in the context of the trivariate meta-analysis of diagnostic test accuracy studies accounting for disease prevalence, is small (one dependence parameter less), but for estimation purposes this is extremely useful given the typically small sample size. 


\section{Small-sample efficiency - Misspecification of the random effects distribution}

An extensive simulation study is conducted (a) to gauge the small-sample efficiency of the ML method, and (b) to investigate in detail the misspecification of the parametric margin or family of copulas of the random effects distribution.

To simulate the data we have used the following generation process: ${ }^{12,37}$

1. Simulate the study size $n$ from a shifted gamma distribution, i.e., $n \sim \operatorname{sGamma}(\alpha=1.2, \beta=$ $0.01, \operatorname{lag}=30)$ and round off to the nearest integer.

2. Simulate $\left(u_{1}, u_{2}, u_{3}\right)$ from a C-vine $C\left(; \tau_{12}, \tau_{13}, \tau_{23 \mid 1}\right)$ via the algorithm in $\mathrm{Joe}^{34} ; \tau^{\prime}$ s are converted to the $\mathrm{BVN}^{38}$, Frank ${ }^{39}$ and rotated Clayton ${ }^{40} \theta$ 's via the relations

$$
\begin{gathered}
\tau=\frac{2}{\pi} \arcsin (\theta), \\
\tau=\left\{\begin{array}{cc}
1-4 \theta^{-1}-4 \theta^{-2} \int_{\theta}^{0} \frac{t}{e^{t}-1} d t \quad, \quad \theta<0 \\
1-4 \theta^{-1}+4 \theta^{-2} \int_{0}^{\theta} \frac{t}{e^{t}-1} d t \quad, \quad \theta>0
\end{array},\right. \\
\tau=\left\{\begin{array}{rr}
\theta /(\theta+2) & , \text { by } 0 \text { or } 180 \text { degrees } \\
-\theta /(\theta+2) & , \text { by } 90 \text { or } 270 \text { degrees }
\end{array},\right.
\end{gathered}
$$

respectively.

3. Convert to beta realizations via $x_{j}=F_{j}^{-1}\left(u_{j}, \pi_{j}, \gamma_{j}\right)$ or normal realizations via $x_{j}=\Phi_{j}^{-1}\left(u_{j}, l\left(\pi_{j}\right), \sigma_{j}\right)$ for $j=1,2$; for the latter convert to proportions via $x_{j}=l^{-1}\left(x_{j}\right)$.

4. Set number of diseased and non-diseased as $n_{1}=n x_{1}$ and $n_{2}=n-n_{1}$, respectively.

5. Set $y_{j}=n_{j} x_{j}$ and then round $y_{j}$ for $j=1,2$.

We randomly generated $B=10^{4}$ samples of size $N=20$ from the C-vine copula mixed model with Clayton blocks rotated by 90 degrees, beta margins, and conditional independence. Table 1 contains the resultant biases, root mean square errors (RMSE), and standard deviations (SD), along with average theoretical SDs $(\sqrt{\bar{V}})$ scaled by $N$, for the MLEs under different block choices and margins assuming conditional at the second level/tree, i.e., we fix $\tau_{23 \mid 1}=0$. The theoretical variances $V$ of the MLEs are obtained via the gradients and the Hessian computed numerically during the maximization process.

Conclusions from the values in the table and ohter computations that we have done are the following:

- ML with the the 'true' vine copula mixed model is highly efficient according to the simulated biases and standard deviations for the meta-analytic parameters of interest. 
Table 1: Small sample of sizes $N=20$ simulations ( $10^{4}$ replications) from the vine copula mixed model with Clayton rotated by 90 degrees blocks, beta margins, and conditional independence, resultant biases, root mean square errors (RMSE) and standard deviations (SD), along with the square root of the average theoretical variances $(\sqrt{\bar{V}})$, scaled by 100 , for the MLEs under different copula choices and margins. Vines have been truncated at the 1 st level/tree, i.e. $\tau_{23 \mid 1}$ is fixed to 0 (conditional independence).

\begin{tabular}{|c|c|c|c|c|c|c|c|c|c|c|}
\hline & Margin & Block & $\pi_{1}=0.8$ & $\pi_{2}=0.7$ & $\pi_{3}=0.4$ & $\gamma_{1}=0.1$ & $\gamma_{2}=0.1$ & $\gamma_{3}=0.05$ & $\tau_{12}=-0.5$ & $\tau_{13}=-0.3$ \\
\hline \multirow[t]{8}{*}{ Bias } & Beta & BVN & 0.04 & 0.00 & 0.02 & -2.28 & -2.01 & -1.11 & -12.24 & -6.17 \\
\hline & & Frank & 0.97 & -1.12 & -0.59 & -2.48 & -1.85 & -1.09 & -14.81 & -7.15 \\
\hline & & Clayton by 90 & 0.07 & 0.06 & 0.05 & -2.52 & -2.14 & -1.18 & -14.66 & -6.35 \\
\hline & & Clayton by 270 & 0.19 & -0.56 & -0.14 & -1.91 & -1.38 & -0.90 & -8.85 & 2.36 \\
\hline & Normal & $\mathrm{BVN}$ & 2.43 & 1.77 & -0.39 & - & - & - & -12.16 & -6.75 \\
\hline & & Frank & 3.19 & 0.52 & -1.00 & - & - & - & -14.57 & -7.45 \\
\hline & & Clayton by 90 & 2.39 & 1.64 & -0.44 & - & - & - & -14.82 & -6.73 \\
\hline & & Clayton by 270 & 2.57 & 1.38 & -0.52 & - & - & - & -8.97 & 1.15 \\
\hline \multirow[t]{8}{*}{ SD } & Beta & BVN & 2.95 & 3.29 & 2.50 & 3.22 & 2.74 & 1.47 & 18.81 & 19.59 \\
\hline & & Frank & 2.99 & 3.70 & 2.69 & 3.11 & 2.86 & 1.50 & 18.82 & 20.53 \\
\hline & & Clayton by 90 & 2.93 & 3.31 & 2.53 & 2.89 & 2.62 & 1.42 & 17.31 & 19.51 \\
\hline & & Clayton by 270 & 3.07 & 3.58 & 2.61 & 3.85 & 3.33 & 1.68 & 27.99 & 24.01 \\
\hline & Normal & BVN & 3.01 & 3.49 & 2.61 & 18.67 & 13.83 & 8.74 & 18.88 & 19.67 \\
\hline & & Frank & 3.16 & 3.84 & 2.80 & 18.78 & 14.10 & 8.87 & 18.86 & 20.55 \\
\hline & & Clayton by 90 & 3.01 & 3.53 & 2.64 & 17.46 & 13.11 & 8.61 & 17.51 & 19.84 \\
\hline & & Clayton by 270 & 3.12 & 3.67 & 2.70 & 21.58 & 16.44 & 9.69 & 27.80 & 23.38 \\
\hline \multirow[t]{8}{*}{$\sqrt{\bar{V}}$} & Beta & BVN & 2.65 & 3.06 & 2.36 & 2.78 & 2.59 & 1.43 & 17.59 & 15.91 \\
\hline & & Frank & 2.63 & 3.22 & 2.35 & 2.42 & 2.68 & 1.43 & 5.21 & 16.60 \\
\hline & & Clayton by 90 & 2.60 & 2.94 & 2.30 & 2.60 & 2.37 & 1.34 & 13.86 & 16.59 \\
\hline & & Clayton by 270 & 2.61 & 3.11 & 2.38 & 2.90 & 2.84 & 1.53 & 14.12 & 14.28 \\
\hline & Normal & BVN & 2.73 & 3.12 & 2.42 & 15.88 & 11.86 & 7.83 & 16.73 & 17.34 \\
\hline & & Frank & 2.66 & 3.30 & 2.42 & 15.97 & 12.75 & 7.95 & 14.93 & 16.76 \\
\hline & & Clayton by 90 & 2.64 & 3.09 & 2.37 & 15.27 & 11.32 & 7.60 & 11.74 & 16.01 \\
\hline & & Clayton by 270 & 2.76 & 3.26 & 2.49 & 16.11 & 13.30 & 8.32 & 15.77 & 17.11 \\
\hline \multirow[t]{8}{*}{ RMSE } & Beta & $\mathrm{BVN}$ & 2.95 & 3.29 & 2.50 & 3.94 & 3.40 & 1.84 & 22.44 & 20.54 \\
\hline & & Frank & 3.14 & 3.87 & 2.76 & 3.97 & 3.40 & 1.85 & 23.95 & 21.74 \\
\hline & & Clayton by 90 & 2.94 & 3.31 & 2.53 & 3.83 & 3.39 & 1.84 & 22.68 & 20.52 \\
\hline & & Clayton by 270 & 3.08 & 3.63 & 2.61 & 4.30 & 3.60 & 1.91 & 29.36 & 24.12 \\
\hline & Normal & BVN & 3.87 & 3.91 & 2.64 & - & - & - & 22.46 & 20.80 \\
\hline & & Frank & 4.49 & 3.88 & 2.97 & - & - & - & 23.84 & 21.86 \\
\hline & & Clayton by 90 & 3.84 & 3.89 & 2.68 & - & - & - & 22.95 & 20.96 \\
\hline & & Clayton by 270 & 4.04 & 3.92 & 2.74 & - & - & - & 29.21 & 23.41 \\
\hline
\end{tabular}


- The MLEs of the meta-analytic parameters are slightly underestimated under copula misspecification. That is, there is some downward bias for these parameters, especially if the "working" model is not close to Kullback-Liebler distance with the "true" model, i.e., it is misspecified. For example in the table there is more bias for the Clayton rotated by 270 degrees and Frank blocks since they have different tail dependence from the 'true' model, i.e., the rotated Clayton by 270 degrees. An interesting result is that the BVN copula performed rather well under misspecification.

- The SDs are rather robust to the copula misspecification.

- The meta-analytic MLEs and SDs are not robust to the margin misspecification, while the MLE of $\tau$ and its SD is.

- Due to small sample size $(N=20)$ there is about $2 \%$ and $10 \%$ bias for the variability and dependence parameters, respectively. These biases decrease as $N$ increases.

The effect of misspecifying the copula choice can be seen as minimal for both the univariate parameters and Kendall's tau. However, note that (a) the meta-analytic parameters are a univariate inference, and hence it is the univariate marginal distribution that matters and not the type of the copula, and, (b) as previously emphasized Kendall's tau only accounts for the dependence dominated by the middle of the data (sensitivities and specificities), and it is expected to be similar amongst different families of copulas. However, the tail dependence varies, as explained in Subsection 4.1, and is a property to consider when choosing amongst different families of copulas, and, hence affects the shape of SROC curves, i.e., prediction. SROC will essentially show the effect of different model (random effect distribution) assumptions, since it is an inference that depends on the joint distribution. We refer the interested reader to Nikoloulopoulos ${ }^{12}$ for further details.

\section{Illustrations}

We illustrate the use of the vine copula mixed model for the meta-analysis of diagnostic accuracy studies accounting from disease prevalence by re-analysing the data of two published meta-analyses. ${ }^{41,42}$ These data have been previously analyzed in the trivariate case by Hoyer and Kuss. ${ }^{19}$

We fit the vine copula mixed model for all different permutations, choices of parametric families of copulas and margins. To make it easier to compare strengths of dependence, we convert from $\tau$ to the BVN $^{38}$, Frank ${ }^{39}$ and rotated Clayton ${ }^{40}$ copula parameter $\theta$ via the relations in (11), (12), and (13).

Model selection is often based on information criteria such as AIC and BIC. We adopt one of this criteria, namely the AIC. The discussion below could also apply to other information criteria. By 
using the ML method, the AIC is $-2 \times \log$-likelihood $+2 \times$ (\#model parameters) and a smaller AIC value indicates a better fitting model.

We further compute the Vuong's test ${ }^{43}$ to reveal if any other vine copula mixed model provides better fit than the standard trivariate GLMM as in Nikoloulopoulos. ${ }^{12}$ The Vuong's test is the sample version of the difference in Kullback-Leibler divergence between two models and can be used to differentiate two parametric models which could be non-nested. Assume that we have Models 1 and 2 with parametric densities $f^{(1)}$ and $f^{(2)}$ respectively, with Model 1 being the vine copula mixed model composed by BVN copulas and normal margins, i.e., the standard GLMM. The sample version of the difference in Kullback-Leibler divergence between two models with MLEs $\hat{\boldsymbol{\theta}}^{(1)}, \hat{\boldsymbol{\theta}}^{(2)}$ is

$$
\bar{D}=\sum_{i=1}^{N} D_{i} / N
$$

where $D_{i}=\log \left[\frac{f^{(2)}\left(Y_{1}, Y_{2} ; \hat{\boldsymbol{\theta}}^{(2)}\right)}{f^{(1)}\left(Y_{1}, Y_{2} ; \hat{\boldsymbol{\theta}}^{(1)}\right)}\right]$. Model 1 is the better fitting model if $\bar{D}<0$, and Model 2 is the better fitting model if $\bar{D}>0$. Vuong ${ }^{43}$ has shown that asymptotically under the null hypothesis that Models 1 and 2 have the same parametric densities $f^{(1)}$ and $f^{(2)}$,

$$
z_{0}=\sqrt{N} \bar{D} / s \stackrel{H_{0}}{\sim} \mathcal{N}(0,1)
$$

where $s^{2}=\frac{1}{N-1} \sum_{i=1}^{N}\left(D_{i}-\bar{D}\right)^{2}$.

\subsection{The $\beta$-D-Glucan data}

In this section we apply the copula mixed models to data on the 8 cohort studies in the meta-analysis in Karageorgopoulos et al. ${ }^{41}$. The interest here is to assess $\beta$-D-Glucan as a serum or plasma marker for the presence of invasive fungal infections, where different medical criteria are used as reference standards.

The AICs showed that a vine copula mixed model with rotated Clayton copulas by 0 (for positive dependence) and 90 (for negative dependence) degrees, beta margins, and permutation $\{12,23,13 \mid 2\}$ provides the best fit (Table 2). It is revealed that a vine copula mixed model with the sensitivity, specificity, and disease prevalence on the original scale provides better fit than the GLMM, which models the sensitivity, specificity and disease prevalence on a transformed scale. In fact, from the Vuong's statistic there is enough improvement to get a statistical significant difference ( $p$-value $=$ 0.008).

Due to small sample size in this study we have then fitted all the truncated at level-1 vines copula mixed models with different permutations and choices of parametric families of copulas and margins. 
Table 2: AICs, estimates and standard errors (SE), along with the Vuong's statistics and $p$-values for the $\beta$-D-Glucan data where the dependence is driven by the number of true negatives $Y_{2}$.

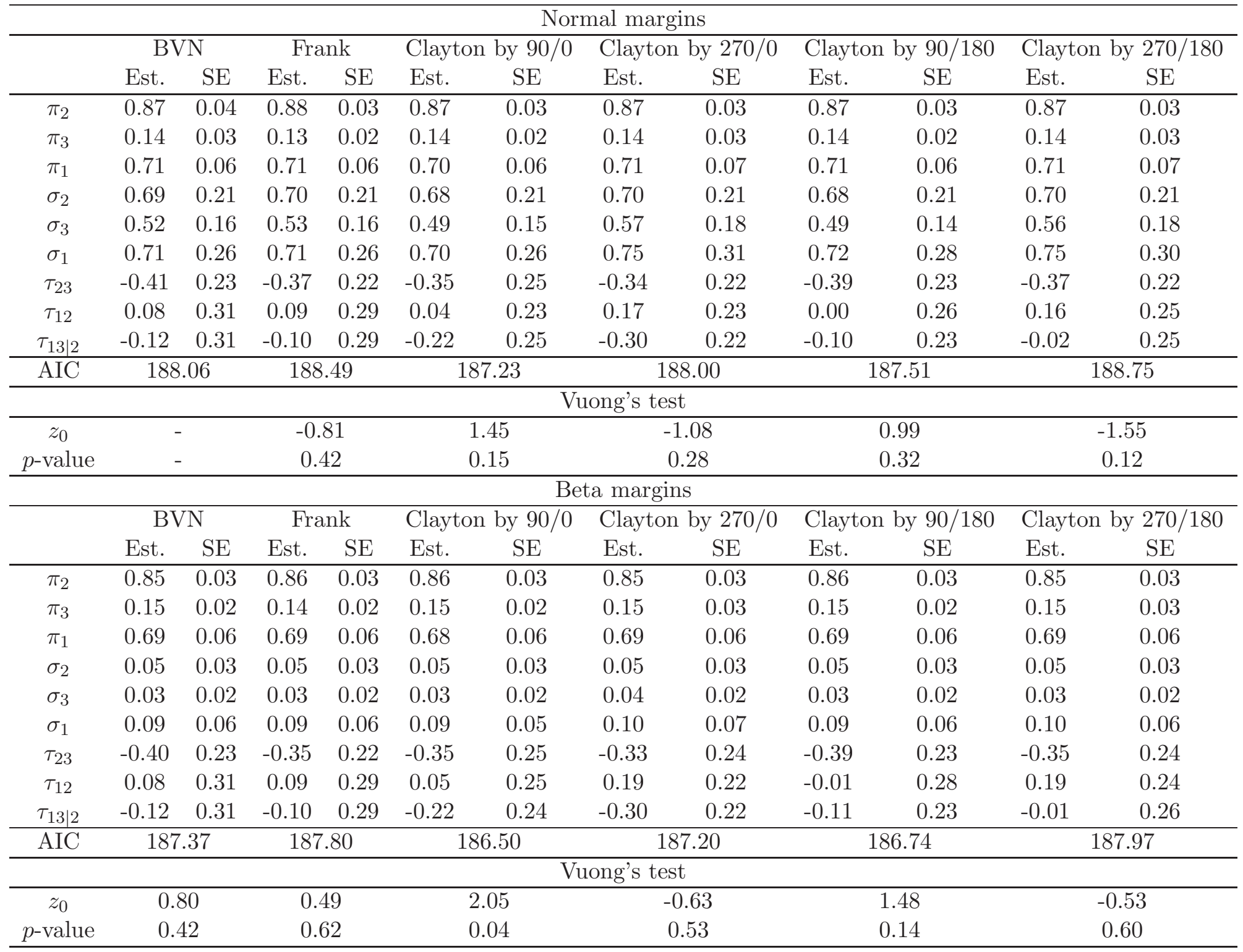


Table 3: AICs, estimates and standard errors (SE), along with the Vuong's statistics and $p$-values for the $\beta$-D-Glucan data where the dependence is driven by the number of diseased persons $Y_{3}$. Vines have been truncated at the 1 st level/tree, i.e. $\tau_{12 \mid 3}$ is fixed to 0 (conditional independence).

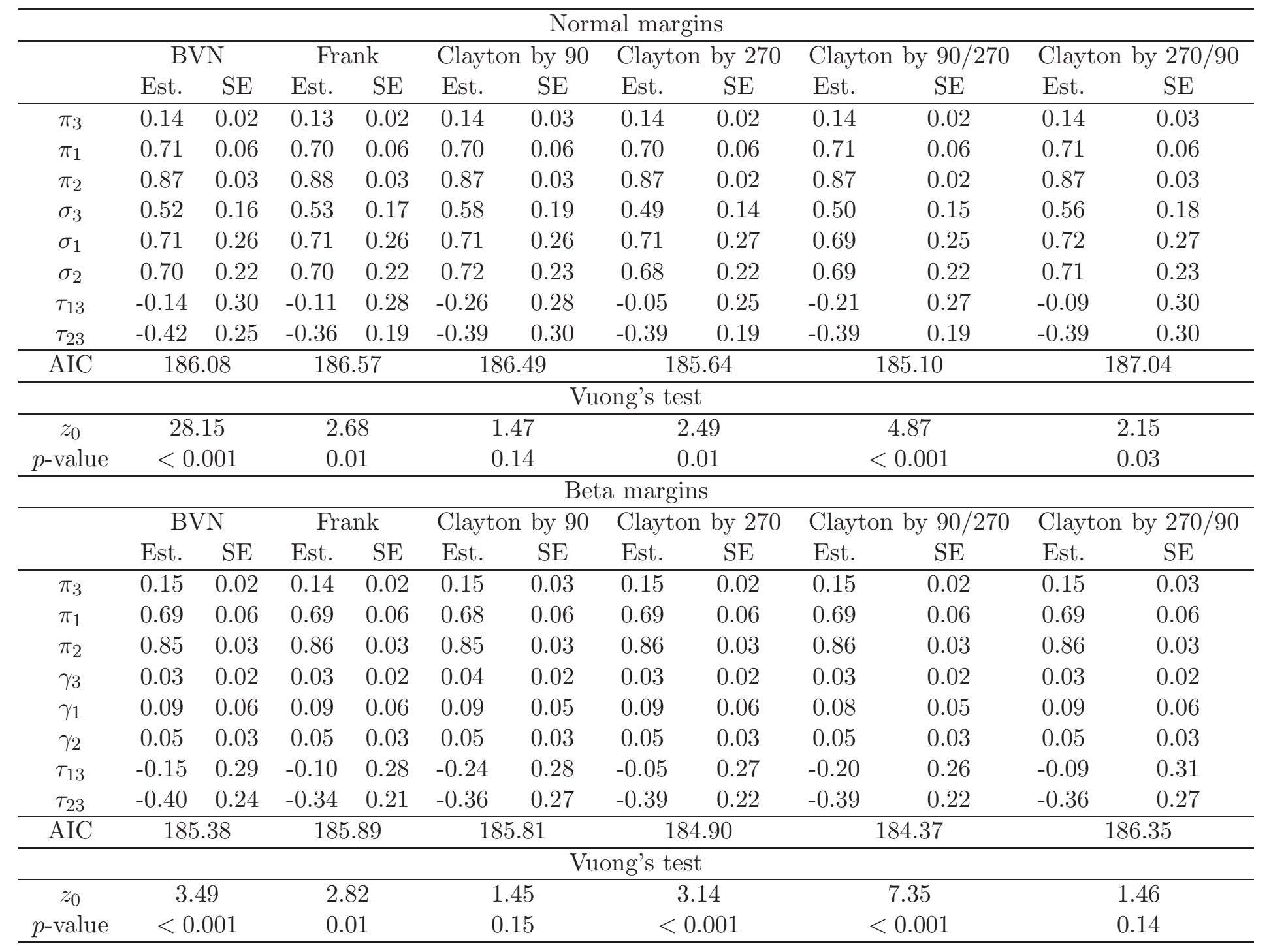


The AICs showed that a vine copula mixed model with rotated Clayton copulas by 90 and 270 (both for negative dependence but with different tail direction) degrees, beta margins, and permutation $\{13,23,12 \mid 3\}$ provides the best fit (Table 3 ). This result suggests some skewness to upper-lower tail for the pair of sensitivity and disease prevalence (i.e., the data display dependence between extreme big values of sensitivity and extreme small values of disease prevalence), and, some skewness to lowerupper tail for the pair of specificity and disease prevalence (i.e., the data display dependence between extreme small values of specificity and extreme big values of disease prevalence).

It is revealed that a vine copula mixed model with the sensitivity, specificity, and prevalence on the original scale provides better fit than the GLMM, which models the sensitivity, specificity and prevalence on a transformed scale. In fact, from the Vuong's statistic there is enough improvement to get a highly statistical significant difference $(p$-value $<0.001)$. In order to include a penalty for the different number of parameters among the models, we used the adjusted Vuong's ${ }^{43}$ version:

$$
\bar{D}-N^{-1}\{\# \text { Model } 2 \text { parameters - \#Model } 1 \text { parameters }\} .
$$

Finally, note that all models roughly agree on the estimated specificity $\hat{\pi}_{1}$ and prevalence $\hat{\pi}_{3}$, but the estimate $\hat{\pi}_{1}$ of sensitivity is smaller when beta margins are assumed.

\subsection{The oral glucose tolerance data}

Ye et al. ${ }^{42}$ meta-analyzed 10 studies on the oral glucose tolerance test for the diagnosis of diabetes mellitus in patients during acute coronary syndrome hospitalization.

Fitting the vine copula mixed model for all different permutations, choices of parametric families of copulas and margins, the resultant estimate of the one of the dependence parameter in the first tree was close to the right boundary of its parameter space, and the conditional dependence parameter was strongly negative. This is a clear case in which the vine copula model with a full structure provides more dependence structure that it is actually required. This has not been revealed in Hoyer and Kuss ${ }^{19}$ since their approximation method substantially underestimates the dependence parameters. ${ }^{12}$

Hence we fit all the truncated at level-1 vines copula mixed models with different permutations and choices of parametric families of copulas and margins. All models agree on the estimated specificity $\hat{\pi}_{2}$ and prevalence $\hat{\pi}_{3}$ but the estimate of $\hat{\pi}_{1}$ is larger under the standard GLMM. Since the number of parameters is the same between the models, we use the log-likelihood at estimates as a measure for goodness of fit between the models. According to the likelihood principle better fits are provided using permutation $\{12,13,23 \mid 1\}$ and the best is provided by the truncated at level- 1 vine copulas composed with BVN copulas and beta margins (Table 4). The log-likelihood is -86.24 and -85.89 for BVN 
Table 4: Maximised log-likelihoods, estimates and standard errors (SE), along with the Vuong's statistics and $p$-values for the oral glucose tolerance data where the dependence is driven by the number of true positives $Y_{1}$. Vines have been truncated at the 1st level/tree, i.e. $\tau_{23 \mid 1}$ is fixed to 0 (conditional independence).

\begin{tabular}{|c|c|c|c|c|c|c|c|c|c|c|c|c|}
\hline \multicolumn{13}{|c|}{ Normal margins } \\
\hline & \multicolumn{2}{|c|}{ BVN } & \multicolumn{2}{|c|}{ Frank } & \multicolumn{2}{|c|}{ Clayton } & \multicolumn{2}{|c|}{ Clayton by 180} & \multicolumn{2}{|c|}{ Clayton by $0 / 180$} & \multicolumn{2}{|c|}{ Clayton by $180 / 0$} \\
\hline & Est. & $\mathrm{SE}$ & Est. & $\mathrm{SE}$ & Est. & $\mathrm{SE}$ & Est. & SE & Est. & $\mathrm{SE}$ & Est. & SE \\
\hline$\pi_{1}$ & 0.71 & 0.07 & 0.72 & 0.06 & 0.72 & 0.06 & 0.71 & 0.06 & 0.72 & 0.06 & 0.70 & 0.07 \\
\hline$\pi_{2}$ & 0.87 & 0.03 & 0.87 & 0.03 & 0.87 & 0.03 & 0.87 & 0.03 & 0.87 & 0.03 & 0.87 & 0.03 \\
\hline$\pi_{3}$ & 0.15 & 0.04 & 0.15 & 0.04 & 0.15 & 0.04 & 0.15 & 0.04 & 0.15 & 0.04 & 0.15 & 0.04 \\
\hline$\sigma_{1}$ & 0.77 & 0.39 & 0.63 & 0.31 & 0.70 & 0.39 & 0.72 & 0.37 & 0.64 & 0.32 & 0.78 & 0.37 \\
\hline$\sigma_{2}$ & 0.67 & 0.20 & 0.69 & 0.22 & 0.71 & 0.23 & 0.64 & 0.20 & 0.71 & 0.24 & 0.63 & 0.19 \\
\hline$\sigma_{3}$ & 0.82 & 0.23 & 0.80 & 0.22 & 0.82 & 0.24 & 0.80 & 0.22 & 0.80 & 0.22 & 0.84 & 0.24 \\
\hline$\tau_{12}$ & 0.60 & 0.33 & 0.86 & 0.00 & 0.68 & 0.52 & 0.57 & 0.40 & 0.89 & 0.45 & 0.47 & 0.36 \\
\hline$\tau_{13}$ & 0.19 & 0.31 & 0.01 & 0.23 & 0.09 & 0.29 & 0.14 & 0.27 & 0.06 & 0.22 & 0.24 & 0.30 \\
\hline $\log L$ & \multicolumn{2}{|c|}{-86.24} & \multicolumn{2}{|c|}{-86.49} & \multicolumn{2}{|c|}{-86.38} & \multicolumn{2}{|c|}{-86.55} & \multicolumn{2}{|c|}{-86.36} & \multicolumn{2}{|c|}{-86.44} \\
\hline \multicolumn{13}{|c|}{ Vuong's test } \\
\hline$z_{0}$ & \multirow{2}{*}{\multicolumn{2}{|c|}{ - }} & \multicolumn{2}{|c|}{-0.24} & \multirow{2}{*}{\multicolumn{2}{|c|}{$\begin{array}{c}-0.23 \\
0.82\end{array}$}} & \multicolumn{2}{|c|}{-0.55} & \multicolumn{2}{|c|}{-0.13} & \multicolumn{2}{|c|}{-0.36} \\
\hline$p$-value & & & \multicolumn{2}{|c|}{0.81} & & & & & \multicolumn{2}{|c|}{0.90} & \multicolumn{2}{|c|}{0.72} \\
\hline & & & & \multicolumn{4}{|c|}{ Beta margins } & & & & \\
\hline & \multicolumn{2}{|c|}{ BVN } & \multicolumn{2}{|c|}{ Frank } & \multicolumn{2}{|c|}{ Clayton } & Clay & by 180 & Clay & by $0 / 180$ & Clay & by $180 / 0$ \\
\hline & Est. & $\mathrm{SE}$ & Est. & $\mathrm{SE}$ & Est. & SE & Est. & $\mathrm{SE}$ & Est. & $\mathrm{SE}$ & Est. & $\mathrm{SE}$ \\
\hline$\pi_{1}$ & 0.69 & 0.07 & 0.69 & 0.06 & 0.69 & 0.06 & 0.69 & 0.06 & 0.70 & 0.06 & 0.68 & 0.07 \\
\hline$\pi_{2}$ & 0.85 & 0.03 & 0.85 & 0.03 & 0.85 & 0.03 & 0.85 & 0.03 & 0.85 & 0.03 & 0.85 & 0.03 \\
\hline$\pi_{3}$ & 0.18 & 0.04 & 0.18 & 0.04 & 0.18 & 0.04 & 0.18 & 0.04 & 0.18 & 0.04 & 0.17 & 0.04 \\
\hline$\gamma_{1}$ & 0.11 & 0.09 & 0.10 & 0.09 & 0.10 & 0.08 & 0.10 & 0.09 & 0.09 & 0.07 & 0.11 & 0.09 \\
\hline$\gamma_{2}$ & 0.05 & 0.03 & 0.05 & 0.03 & 0.06 & 0.03 & 0.04 & 0.03 & 0.06 & 0.03 & 0.04 & 0.03 \\
\hline$\gamma_{3}$ & 0.07 & 0.04 & 0.07 & 0.04 & 0.08 & 0.04 & 0.07 & 0.04 & 0.07 & 0.04 & 0.08 & 0.04 \\
\hline$\tau_{12}$ & 0.56 & 0.29 & 0.63 & 0.40 & 0.60 & 0.30 & 0.52 & 0.36 & 0.73 & 0.41 & 0.43 & 0.35 \\
\hline$\tau_{13}$ & 0.21 & 0.29 & 0.11 & 0.34 & 0.14 & 0.26 & 0.15 & 0.28 & 0.08 & 0.22 & 0.26 & 0.30 \\
\hline $\log L$ & $-8 !$ & & & & -86 & & & & & & & .09 \\
\hline & & & & & & & long's & & & & & \\
\hline$z_{0}$ & & & & & 0 & & & & & & & 20 \\
\hline$p$-value & & & & & & & & & & & & 85 \\
\hline
\end{tabular}


building block with normal and beta margins, respectively; thus a beta margin seems to be a better fit for the data. However, according to the Vuong's test the the truncated at level-1 vine copula mixed model with beta margins does not provides better fit $(p$-value $=0.574)$ than a model with normal margins. The latter is close the the standard GLMM.

\section{Discussion}

We have proposed a vine copula mixed model for trivariate meta-analysis of diagnostic test accuracy studies accounting for disease prevalence. Our general statistical model allows for selection of a bivariate block independently among a variety of parametric copula families, i.e., there are no constraints in the choices of bivariate parametric families of copulas.

It also includes the trivariate GLMM as a special case and can be seen to provide an improvement over the GLMM on the basis of the log-likelihood and Vuong's ${ }^{43}$ statistic. Hence, superior statistical inference for the meta-analytic parameters of interest can be achieved. This improvement relies on the fact that the random effects distribution is expressed via copulas which can provide a wide range of flexible tail dependencies and asymmetries. ${ }^{16}$ The MVN assumption is used in statistics because it is mathematically convenient, but one of our points is that the normality assumption is not reasonable if there is some skewness in the joint tails. The theory and application of copulas have become important in finance, insurance and other areas, in order to deal with dependence in the joint tails. Here, we have indicated that this can also be important in meta-analysis of diagnostic test accuracy studies.

It has been reported in the literature that since the trivariate GLMM has three correlation parameters, there might be estimation problems relating to the correlation parameters, particularly if the sample size is small. ${ }^{8}$ Here we use the notion of "truncated at level-1 vine copulas", which allow both parsimony and flexible dependence structure, to subside this problem. This is due to the main result in Joe et al. ${ }^{16}$ : all bivariate margins of the vine copula have upper/lower tail dependence if the bivariate copulas at level 1 have upper/lower tail dependence. In a copula modelling framework as well as in any other statistical modelling framework, the pursuit of perfection is illusory and a balance should always be struck between fit and parsimony. ${ }^{44}$

A challenging direction of future research is to extend our framework to model data from studies that do not report all outcomes via pattern mixture models. Pattern mixture models are studied in Shen and Weissfeld ${ }^{45}$ for multivariate copulas and in Mavridis et al. ${ }^{46}$ for multivariate meta-analysis.

\section{Software}

$\mathrm{R}$ functions to implement the vine copula mixed model for meta-analysis of diagnostic test accuracy studies acounting for disease prevalence are part of the $\mathrm{R}$ package CopulaREMADA. ${ }^{47}$ 


\section{References}

[1] Jackson D, Riley R, White IR. Multivariate meta-analysis: Potential and promise. Statistics in Medicine. $2011 ; 30(20): 2481-2498$.

[2] Mavridis D, Salanti G. A practical introduction to multivariate meta-analysis. Statistical Methods in Medical Research. 2013;22(2):133-158.

[3] Ma X, Nie L, Cole SR, Chu H. Statistical methods for multivariate meta-analysis of diagnostic tests: An overview and tutorial. Statistical Methods in Medical Research. 2013;DOI:10.1177/0962280213492588.

[4] Chu H, Nie L, Chen Y, Huang Y, Sun W. Bivariate random effects models for meta-analysis of comparative studies with binary outcomes: Methods for the absolute risk difference and relative risk. Statistical Methods in Medical Research. 2012;21(6):621-633.

[5] Chu H, Cole SR. Bivariate meta-analysis of sensitivity and specificity with sparse data: a generalized linear mixed model approach. Journal of Clinical Epidemiology. 2006;59(12):1331-1332.

[6] Arends LR, Hamza TH, van Houwelingen JC, Heijenbrok-Kal MH, Hunink MGM, Stijnen T. Bivariate Random Effects Meta-Analysis of ROC Curves. Medical Decision Making. 2008;28(5):621-638.

[7] Hamza TH, Arends LR, van Houwelingen HC, Stijnen T. Multivariate random effects meta-analysis of diagnostic tests with multiple thresholds. BMC Medical Research Methodology. 2009;9(1):1-15.

[8] Chu H, Nie L, Cole SR, Poole C. Meta-analysis of diagnostic accuracy studies accounting for disease prevalence: Alternative parameterizations and model selection. Statistics in Medicine. 2009;28(18):2384-2399.

[9] Brenner H, Gefeller O. Variation of sensitivity, specificity, likelihood ratios and predictive values with disease prevalence. Statistics in Medicine. 1997;16(9):981-991.

[10] Leeflang MMG, Bossuyt PMM, Irwig L. Diagnostic test accuracy may vary with prevalence: implications for evidence-based diagnosis. Journal of Clinical Epidemiology. 2009;62(1):5-12.

[11] Leeflang MMG, Rutjes AWS, Reitsma JB, Hooft L, Bossuyt PMM. Variation of a test's sensitivity and specificity with disease prevalence. Canadian Medical Association Journal. 2013;185(11):E537-E544.

[12] Nikoloulopoulos AK. A mixed effect model for bivariate meta-analysis of diagnostic test accuracy studies using a copula representation of the random effects distribution. ArXiv e-prints. 2015;arXiv:1502.07505.

[13] Joe H. Multivariate Models and Dependence Concepts. London: Chapman \& Hall; 1997.

[14] McNeil AJ, Nešlehová J. Multivariate Archimedean copulas, $D$-monotone functions and $l_{1}$-norm symmetric distributions. Annals of Statistics. 2009;37:3059-3097.

[15] Nikoloulopoulos AK, Joe H, Chaganty NR. Weighted scores method for regression models with dependent data. Biostatistics. 2011;12:653-665.

[16] Joe H, Li H, Nikoloulopoulos AK. Tail dependence functions and vine copulas. Journal of Multivariate Analysis. 2010;101:252-270.

[17] Kurowicka D, Joe H. Dependence Modeling - Handbook on Vine Copulae. Singapore: World Scientific Publishing Co; 2011.

[18] Joe H. Dependence Modeling with Copulas. London: Chapman \& Hall; 2014.

[19] Hoyer A, Kuss O. Meta-analysis of diagnostic tests accounting for disease prevalence: A new model using trivariate copulas. Statistics in Medicine. 2015;34(11):1912-1924.

[20] Kuss O, Hoyer A, Solms A. Meta-analysis for diagnostic accuracy studies: a new statistical model using betabinomial distributions and bivariate copulas. Statistics in Medicine. 2014;33(1):17-30.

[21] Demidenko E. Mixed Models: Theory and Applications. Hoboken, New Jersey: John Wiley \& Sons; 2004.

[22] Nelsen RB. An Introduction to Copulas. New York: Springer-Verlag; 2006.

[23] Sklar M. Fonctions de répartition à $n$ dimensions et leurs marges. Publications de l'Institut de Statistique de l'Université de Paris. 1959;8:229-231.

[24] Nikoloulopoulos AK, Joe H. Factor copula models for item response data. Psychometrika. 2015;80:126-150.

[25] Nikoloulopoulos AK, Joe H, Li H. Vine copulas with asymmetric tail dependence and applications to financial return data. Computational Statistics \& Data Analysis. 2012;56:659-3673.

[26] Joe H. Families of $m$-variate distributions with given margins and $m(m-1) / 2$ bivariate dependence parameters. In: Rüschendorf L, Schweizer B, Taylor MD, editors. Distributions with Fixed Marginals and Related Topics. vol. 28. Hayward, CA: Institute of Mathematical Statistics; 1996. p. 120-141.

[27] Aas K, Czado C, Frigessi A, Bakken H. Pair-copula constructions of multiple dependence. Insurance: Mathematics \& Economics. 2009;44:182-198.

[28] Nikoloulopoulos AK. On the estimation of normal copula discrete regression models using the continuous extension and simulated likelihood. Journal of Statistical Planning and Inference. 2013;143:1923-1937.

[29] Nikoloulopoulos AK. Efficient estimation of high-dimensional multivariate normal copula models with discrete spatial responses. Stochastic Environmental Research and Risk Assessment. 2015;DOI:10.1007/s00477-015-1060-2 .

[30] Panagiotelis A, Czado C, Joe H. Pair Copula Constructions for Multivariate Discrete Data. Journal of the American Statistical Association. 2012;107:1063-1072.

[31] Nash JC. Compact Numerical Methods for Computers: Linear Algebra and Function Minimisation. New York: 
Hilger; 1990. 2nd edition.

[32] Serfling RJ. Approximation Theorems of Mathematical Statistics. New York: Wiley; 1980.

[33] Stroud AH, Secrest D. Gaussian Quadrature Formulas. Englewood Cliffs, NJ: Prentice-Hall; 1966.

[34] Joe H. Dependence comparisons of vine copulae with four or more variables. In: Kurowicka D, Joe H, editors. Dependence Modeling: Handbook on Vine Copulae. Singapore: World Scientific; 2011. p. 139-164.

[35] Hua L, Joe H. Tail order and intermediate tail dependence of multivariate copulas. Journal of Multivariate Analysis. 2011;102(10):1454-1471.

[36] Nikoloulopoulos AK, Karlis D. Copula model evaluation based on parametric bootstrap. Computational Statistics \& Data Analysis. 2008;52:3342-3353.

[37] Paul M, Riebler A, Bachmann LM, Rue H, Held L. Bayesian bivariate meta-analysis of diagnostic test studies using integrated nested Laplace approximations. Statistics in Medicine. 2010;29(12):1325-1339.

[38] Hult H, Lindskog F. Multivariate extremes, aggregation and dependence in elliptical distributions. Advances in Applied Probability. 2002;34:587-608.

[39] Genest C. Frank's family of bivariate distributions. Biometrika. 1987;74(3):549-555.

[40] Genest C, MacKay J. The joy of copulas: bivariate distributions with uniform marginals. The American Statistician. 1986;40(4):280-283.

[41] Karageorgopoulos DE, Vouloumanou EK, Ntziora F, Michalopoulos A, Rafailidis PI, Falagas ME. $\beta$-D-Glucan assay for the diagnosis of invasive fungal infections: a meta-analysis. Clinical Infectious Diseases. 2011;52(6):750-770.

[42] Ye Y, Xie H, Zhao X, Zhang S. The oral glucose tolerance test for the diagnosis of diabetes mellitus in patients during acute coronary syndrome hospitalization: a meta-analysis of diagnostic test accuracy. Cardiovascular Diabetology. $2012 ; 11(5): 155$.

[43] Vuong QH. Likelihood Ratio Tests for Model Selection and Non-Nested Hypotheses. Econometrica. 1989;57(2):pp. 307-333.

[44] Genest C, Favre AC. Everything you always wanted to know about copula modeling but were afraid to ask. Journal of Hydrologic Engineering. 2007;12:347-368.

[45] Shen C, Weissfeld L. A copula model for repeated measurements with non-ignorable non-monotone missing outcome. Statistics in Medicine. 2006;25(14):2427-2440.

[46] Mavridis D, White IR, Higgins JPT, Cipriani A, Salanti G. Allowing for uncertainty due to missing continuous outcome data in pairwise and network meta-analysis. Statistics in Medicine. 2014;34(5):721-741.

[47] Nikoloulopoulos AK. CopulaREMADA: Copula random effects model for bivariate meta-analysis of diagnostic test accuracy studies; 2015. Available from: http://CRAN.R-project.org/package=CopulaREMADA. 